\title{
ORIGINAL ARTICLE \\ A subtype of multiple sclerosis defined by an activated immune defense program
}

\author{
LGM van Baarsen¹, TCTM van der Pouw Kraan ${ }^{1}$, JJ Kragt ${ }^{2}$, JMC Baggen ${ }^{1}$, F Rustenburg1, T Hooper ${ }^{1}$, \\ JF Meilof ${ }^{3}$, MJ Fero ${ }^{4}$, CD Dijkstra ${ }^{1}$, CH Polman ${ }^{2}$ and CL Verweij ${ }^{1}$ \\ ${ }^{1}$ Department of Molecular Cell Biology \& Immunology, VU Medical Center, Amsterdam, The Netherlands; ${ }^{2}$ Department of Neurology, \\ VU Medical Center, Amsterdam, The Netherlands; ${ }^{3}$ Department of Neurology, Martini Ziekenhuis, Groningen, The Netherlands \\ and ${ }^{4}$ Stanford Functional Genomics Facility, Stanford School of Medicine, Stanford, CA, USA
}

\begin{abstract}
Given the heterogeneous nature of multiple sclerosis (MS), we applied DNA microarray technology to determine whether variability is reflected in peripheral blood (PB) cells. In this study, we studied whole-blood gene expression profiles of 29 patients with relapsing-remitting MS (RRMS) and 25 age- and sex-matched healthy controls. We used microarrays with a complexity of $43 \mathrm{~K}$ cDNAs. The data were analyzed using sophisticated pathway-level analysis in order to provide insight into the deregulated peripheral immune response programs in MS. We found a remarkable elevated expression of a spectrum of genes known to be involved in immune defense in the PB of MS patients compared to healthy individuals. Cluster analysis revealed that the increased expression of these genes was characteristic for approximately half of the patients. In addition, the gene signature in this group of patients was comparable with a virus response program. We conclude that the transcriptional signature of the PB cells reflects the heterogeneity of $M S$ and defines a sub-population of RRMS patients, who exhibit an activated immune defense program that resembles a virus response program, which is supportive for a link between viruses and $M S$.
\end{abstract}

Genes and Immunity (2006) 7, 522-531. doi:10.1038/sj.gene.6364324; published online 13 July 2006

Keywords: heterogeneity; multiple sclerosis; microarray; interferon; genomics; autoimmunity

\section{Introduction}

Multiple sclerosis (MS) is a chronic neurological disorder in which demyelination and inflammation occur in the white matter of the central nervous system. ${ }^{1}$ The disease has a heterogeneous nature, which is reflected in the clinical presentation, ranging from mild-to-severe demyelinating disease. The wide variation in response to treatment in MS is consistent with the heterogeneous nature of the disease. For example, treatment with interferon (IFN)- $\beta$ exhibits only partial responses in a subgroup of MS patients, ${ }^{2}$ suggesting that distinct disease mechanisms are at play in MS.

The heterogeneous nature of MS is also reflected by immunopathological studies using post-mortem brain material from MS patients, which revealed profound heterogeneity in the patterns of demyelination between different patients, whereas within the same patient different active plaques were similar. ${ }^{3}$ Recently, the heterogeneity of MS was also dissected in vivo using non-invasive magnetic resonance imaging (MRI) techniques combined with statistical remodeling. ${ }^{4}$ Although in

Correspondence: Professor CL Verweij, Department of Molecular Cell Biology \& Immunology, VU Medical Center, PO Box 7057, 1007 MB Amsterdam, The Netherlands.

E-mail: c.verweij@vumc.nl

Received 24 April 2006; revised 9 June 2006; accepted 11 June 2006; published online 13 July 2006 this study the MRI data could not be correlated with pathological data, the observed subgroups were clinically meaningful. The existence of heterogeneity in the brain of MS patients is proposed to reflect different pathogenic processes underlying MS: ${ }^{3}$ an inflammation-mediated and an immune-independent demyelinating form, which both ultimately might drive brain tissue destruction. Thus, different disease mechanisms may vary among patients and, perhaps, in different stages of disease.

The heterogeneity most likely has its origin in the multifactorial nature of the disease, whereby specific combinations of environmental factors and a varying polygenic background are likely to influence not only susceptibility but also the severity and disease outcome.

As the concordance among genetically identical twins is less than complete, an environmental factor or factors are anticipated to have a major role in the risk of developing MS in a genetically susceptible host. ${ }^{5}$ Findings from epidemiological studies, which revealed an uneven distribution of MS with disease more prevalent in temperate climates ${ }^{6}$ as well as data from migration studies, implicate an important role for an infectious agent in MS etiology. ${ }^{7}$ A number of viruses have been associated with the development of MS. ${ }^{8}$ However, despite this knowledge, the environmental factor(s) that contribute to MS remain to be determined.

A powerful way to provide insight into the complexity and pathogenesis of MS has arisen from DNA microarray technology, which provides the opportunity to 
determine differences in gene expression of a large portion of the genome in search of genes that are differentially expressed between patients with clinically diagnosed relapsing-remitting MS (RRMS). By largescale gene expression profiling in blood cells from patients with MS and healthy controls, one can obtain a molecular portrait that is disease associated. This molecular portrait typically represents the contributions and interactions of numerous distinct cells and diverse factors that are associated with disease. In addition, a systematic analysis of the gene expression profiles should reveal whether the heterogeneous nature of MS is reflected in the PB cells. This type of analysis will not only disclose markers that are indicative for differential disease mechanisms in MS, but may, in the case of a pathogenic stimulus of host cells, reveal a pathogeninduced transcriptional program.

\section{Results}

Differential gene expression in PB of RRMS patients compared to healthy controls

We studied differences in gene expression in PB cells derived from healthy controls and patients with RRMS. A schematic outline of the data analysis is shown in Figure 1. At first, the data were analyzed as two-class, unpaired data using significance analysis of microarrays (SAM). ${ }^{9}$ A total of 2205 genes were selected whose transcript levels showed significantly differential expression between patients and controls. Of these genes, 1059 were significantly upregulated, whereas 966 genes were downregulated in RRMS (Figure 2a).

The genes with the most upregulated ( $>1.3$-fold) expression levels encode for immunoglobulin-related proteins, for example, immunoglobulin lambda constant 2 chain, immunoglobulin lambda variable group and immunoglobulin kappa constant chain. Other genes with marked increased expression levels in the PB cells of MS patients belonged to a group of IFN-induced genes, for example, IFN-induced protein 44 (IFI44L), IFN-induced transmembrane protein 1 (9-27; IFITM1), IFN- $\alpha$-inducible protein (clone IFI-15K; G1P2), IFN-induced transmembrane protein 3 (1-8U; IFITM3), myxovirus (influenza virus) resistance 1, IFN-inducible protein $\mathrm{p} 78$ (mouse; Mx1), IFN-induced transmembrane protein

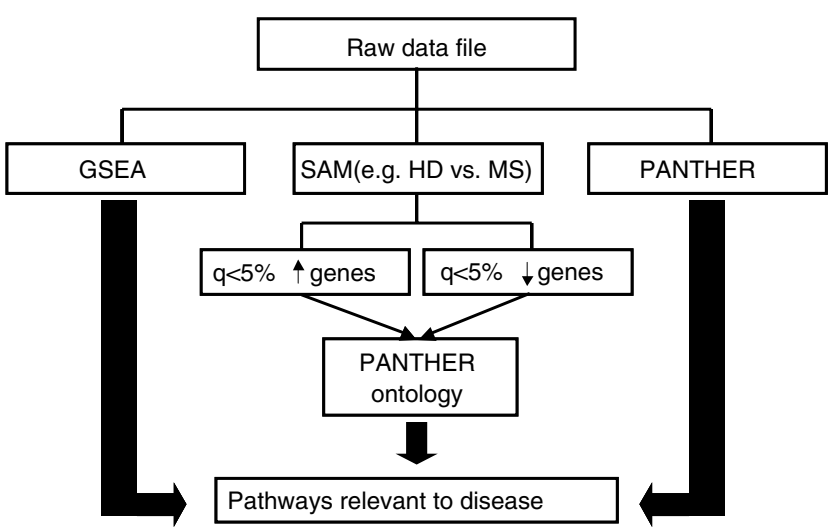

Figure 1 Flowchart data analysis. This figure shows our data analysis steps from raw microarray data toward defining pathways relevant for disease.
2 (1-8D; IFITM2), adenosine deaminase, RNA-specific (ADAR1), IFN-regulatory factor 2 (IRF2), IRF7, 2'-5'oligoadenylate synthetase 2, 69/71 kDa (OAS2) and others. Of note were also the increased levels of the antioxidant enzyme glutathione peroxidase 1 (GPX1) and the NADPH oxidase neutrophil cytosolic factor 1 (NCF1) that are involved in the oxidative stress response of the cell.

The top 10 of the most downregulated genes encode for TAF9 RNA polymerase II (TAF9), catalase (CAT), myomesin (M-protein) 2 (MYOM2), CREBBP/EP300 inhibitor 1 (CRI1), Zwilch (FLJ10036), T-cell leukemia/ lymphoma 1A (TCL1A), short coiled-coil protein (SCOC), hemoglobin, gamma G (HBG2), claudin 12 (CLDN12), GrpE-like 2 and mitochondrial (Escherichia coli; GRPEL2), which are involved in several different processes.

Ontology analysis of significantly differential expressed genes To categorize systematically the 2025 significantly differential expressed genes into functional groups, we applied the Protein ANalysis THrough Evolutionary Relationships Classification System (PANTHER) ontology classification system (Applied Biosystems, Foster City, CA, USA). PANTHER uses the binomial statistics tool $^{10}$ to compare our gene list to a reference list (NCBI: Homo sapiens genes) to determine the statistically significant over-representation of functional groups of genes. The differentially upregulated genes represented 17 significant ontology/functional groups. These are: Alzheimer's disease, presenilin signaling, JAK/STAT signaling, Fas signaling, IFN- $\gamma$ signaling, inflammationmediated immunity, apoptosis signaling, angiogenesis, Parkinson disease, interleukin signaling, heteromeric G-protein signaling pathway-rod outer segment phototransduction, metabotropic glutamate receptor group III biology, ionotrophic glutamate receptor biology, ubiquitin proteasome signaling, D2/D3/D4 dopamine receptor-mediated signaling, Toll-like receptor (TLR) signaling and cysteine biosynthesis (Figure 2b). Analysis of the downregulated gene list revealed two significant categories: p53 signaling and again ubiquitin proteasome signaling (Figure 2c). As this analysis revealed that both the significantly upregulated genes as well as the downregulated genes could be grouped into ubiquitin proteasome signaling, we took a detailed look at the individual genes that constitute these categories. This revealed that most of the upregulated ubiquitin proteasome-related genes encoded proteins with ubiquitinconjugating activities to mark cellular proteins for degradation by the proteasome. In contrast, the downregulated ones represent genes that encode proteasome subunits and the UCHL5 gene encoding a deubiquitinating enzyme. Hence, the differential regulation of genes involved in ubiquitination is compatible with an increased ubiquitin proteasome protein degradation activity.

Pathway-level analysis of differentially expressed genes Next, we performed a pathway-level analysis, which is based on the premise that subtle coordinate changes in gene expression levels across the whole network of genes within a certain pathway can already have major effects on the outcome. At the individual gene level, these gene expression changes do not necessarily have to be 


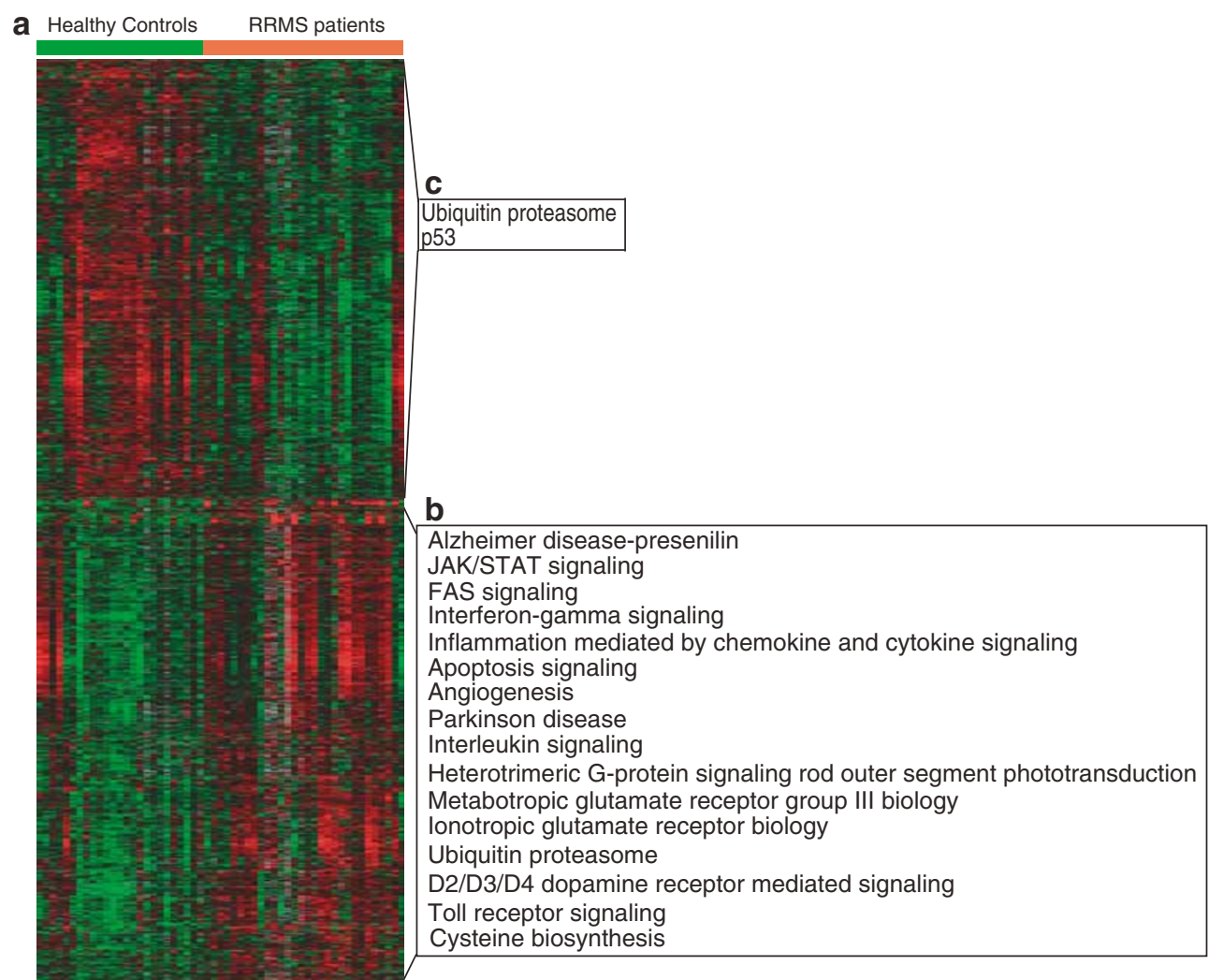

Figure 2 Statistical analysis combined with PANTHER's ontology. (a) Supervised cluster analysis of significantly differential expressed genes. A total of 2025 significantly differential expressed genes (FDR $<5 \%$ ) were selected after SAM analysis between RRMS patients and healthy controls. These genes were supervised (one-way) hierarchical clustered and visualized by Treeview. This type of clustering will place genes that show a correlated expression level in adjacent rows. Each column represents the data of one array and each row shows the relative expression levels of a single gene for all samples. Red color means a relative higher expression level than the median expression level across all samples. Green color means a relative lower expression level than the median expression level across all samples. Black color indicates that the expression level is equal to the median expression level and gray represents missing values. (b) PANTHER ontology analysis on the upregulated genes. Using the PANTHER ontology analysis, the significantly upregulated genes could be classified into different functional categories. (c) PANTHER ontology analysis on the downregulated genes.

significantly different. In order to find such coordinated shifts of gene expression values, we applied the PANTHER $^{11}$ and Gene Set Enrichment Analysis $(\mathrm{GSEA})^{12}$ pathway-level algorithms that are based on the usage of the whole array data set.

Application of PANTHER pathway-level analysis, which is restricted to pathway sets from PANTHER resources, revealed nine pathways within the RRMS patient group (Table 1). These results confirmed the activation of genes involved in inflammation, IFN- $\gamma$ signaling, Alzheimer disease, JAK-STAT signaling, TLR signaling and the metabotropic glutamate receptor group III pathway, all of which were also observed in the PANTHER ontology analysis (Figure 2). In addition, this analysis revealed the coordinated upregulation of genes involved in glycolysis and (nor)adrenaline biosynthesis and the downregulation of genes involved in transcriptional regulation. These data indicate that the observed host response profile in RRMS represents the concerted action of multiple transcriptional regulators, of which a part may reflect TLR-induced innate immune activation.

We confirmed these results using the recently available GSEA pathway-analysis algorithm, ${ }^{12}$ which has the advantage that one can choose which gene sets to use for the analysis, including custom-designed gene sets (data not shown). The data from these two approaches
Table 1 PANTHER pathway-level analysis: RRMS patients versus healthy controls

\begin{tabular}{|c|c|c|}
\hline $\begin{array}{l}\text { Pathway coordinately changed in } \\
\text { RRMS }\end{array}$ & $\begin{array}{l}\text { Up/down in } \\
\text { RRMS }\end{array}$ & $\begin{array}{l}\text { In PANTHER } \\
\text { ontology list }\end{array}$ \\
\hline $\begin{array}{l}\text { Inflammation mediated by } \\
\text { chemokine and cytokine } \\
\text { signaling pathway }\end{array}$ & Up & Yes \\
\hline $\begin{array}{l}\text { Alzheimer disease - presenilin } \\
\text { pathway }\end{array}$ & Up & Yes \\
\hline IFN- $\gamma$ signaling pathway & Up & Yes \\
\hline Toll-receptor signaling pathway & $\mathrm{Up}$ & Yes \\
\hline JAK/STAT signaling pathway & Up & Yes \\
\hline $\begin{array}{l}\text { Metabotropic glutamate receptor } \\
\text { group III pathway }\end{array}$ & Up & Yes \\
\hline General transcription regulation & Down & No \\
\hline Glycolysis & Up & No \\
\hline $\begin{array}{l}\text { Adrenaline and noradrenaline } \\
\text { biosynthesis }\end{array}$ & Up & No \\
\hline
\end{tabular}

Abbreviations: IFN, interferon; RRMS, relapsing-remitting multiple sclerosis.

PANTHER pathway-level analysis was used to define those pathways of which the corresponding gene expression levels are coordinately changed throughout a significant part of the pathway. Pathways were considered significantly changed with a $P$-value $<0.05$. 
unanimously demonstrate the occurrence of an upregulated expression of genes involved in IFN signaling. The significantly differential expression of the IFN response program was confirmed by quantitative real-time polymerase chain reaction analysis of the IFN-regulated genes MxA, IFITM1 and G1P2 (data not shown). Hence, pathway-level analyses reveal that RRMS PB cells harbor features that are indicative for immune activation of TLRs possibly by microbial agents, leading to increased expression of IFNs.

Comparison of MS host response program with the common pathogen response program

In order to create a framework for a more detailed comparison of the RRMS-specific pathways with those of a microbial response program, we used data from a meta-analysis of gene expression profiles after microbial infection. ${ }^{13}$ In this analysis, the transcriptional profile of 32 in vitro studies that involved 77 different hostpathogen interactions have been analyzed together, which resulted in a defined common host transcriptional response program. In order to analyze which pathways are represented in the common response program, we applied PANTHER ontology analysis (Table 2). This analysis revealed a total of 29 significant pathways in the common pathogen response program. Comparison of these pathways with the ones we identified in RRMS showed that there is a significant ( $\chi^{2}$ test; $P=0.03$ ) overlap. Of the 20 RRMS-specific pathways, 11 are present within the common host response program, suggesting that the RRMS profile may reflect, in part, a response to a microbial infection.

\section{Selective upregulation of the type-I IFN signaling pathway in RRMS}

IFNs, which are best known for their anti-viral properties, ${ }^{14}$ are grouped into two categories, type-I and type-II IFNs. Type-I IFNs are mainly produced directly after viral infection, whereas type-II IFNs are secondary produced by activated $\mathrm{T}$ and NK cells. Moreover, although type-I and type-II IFN response programs share a number of genes, their activities can be distinguished based on a number of differentially activated response genes. Thus, a detailed analysis of the observed IFN-induced genes in RRMS is likely to disclose information on the inducing type of IFN. Therefore, we obtained small but specific type-I IFN (IFN- $\alpha$; 9 genes) and type-II IFN (IFN- $\gamma$; six genes) gene sets from the Biocarta database (www.biocarta.com; Supplementary data). To investigate the relative contribution of either gene set in the RRMS gene expression profile, we calculated for each gene set the mean gene expression level ( $\log 2$ ratio) per patient and healthy control, and compared the two groups with each other (Figure 3). This analysis showed that the mean gene expression level of the type-I IFN gene set was significantly higher in the MS patient group when compared to the healthy control group. The mean gene expression level of the type-II IFN genes was similar between patients and controls. Hence, we conclude that type-I IFNs are induced, which can cause a positive feedback loop resulting in more expression of type-I IFNinduced genes.

As our data revealed a deregulated expression of genes involved in innate immunity, like TLR and type-I
Table 2 Deregulated pathways in RRMS partly overlap with a common pathogen response pathway

Significant upregulated pathways in common MS versus $H D$ pathogen response ${ }^{13}$

Inflammation mediated by chemokine and $\mathbf{X}$

cytokine signaling pathway

Apoptosis signaling pathway

Toll-receptor signaling pathway

Interleukin signaling pathway

T-cell activation

Angiogenesis

B-cell activation

Oxidative stress response

EGF receptor signaling pathway

FAS signaling pathway

TGF- $\beta$ signaling pathway

PDGF signaling pathway

Integrin signaling pathway

Parkinson disease

Axon guidance mediated by semaphorins

Heterotrimeric G-protein signaling pathway-Gq

$\alpha$ - and Go $\alpha$-mediated pathway

Alzheimer disease - presenilin pathway

Plasminogen-activating cascade

Blood coagulation

JAK/STAT signaling pathway

FGF signaling pathway

Cadherin signaling pathway

Purine metabolism

Ras pathway

Wnt signaling pathway

Heterotrimeric G-protein signaling pathway-Gi

$\alpha$ - and Gs $\alpha$-mediated pathway

Glycolysis

Endothelin signaling pathway

IFN- $\gamma$ signaling pathway

$\mathrm{X}$

$\mathrm{X}$

$\mathbf{X}$

$\mathbf{X}$

$\mathrm{X}$

$x$

$\mathbf{X}$

$\mathbf{X}$

Abbreviations: EGF, epidermal growth factor; FGF, fibroblast growth factor; HD, healthy donores; IFN, interferon; MS, multiple sclerosis; PDGF, platelet-derived growth factor; RRMS, relapsingremitting multiple sclerosis; TGF, transforming growth factor.

The common pathogen response genes described by Jenner and Young were classified into functional categories using the PANTHER's classification system. A comparison was made between the common pathogen response program and the deregulated pathways we found in RRMS. The overlapping pathways are given in bold and marked with an X. A significant part $\left(P=0.03 ; \chi^{2}\right.$ test) of the RRMS pathways overlap with the common pathogen response.

IFN response genes, we studied the innate response program in more detail. Further insight into the regulatory components of the gene modules that are activated in RRMS is likely to disclose information about the stimulating component. Genes that are most strongly upregulated via TLRs are nuclear factor (NF) $-\kappa \mathrm{B}$ response genes via the MYD88-dependent route and IFN-stimulated genes (ISGs) via the transcription factors IRF3 and IRF7. ${ }^{15}$ Although activation of different TLRs upregulate many of the same genes, these receptors also mediate stimulus-specific responses. Genes that are strongly upregulated via TLR4 are the ISGs and NF- $\kappa \mathrm{B}$ response genes. In contrast, the TLR3, TLR7/TLR8 and TLR9 induce a pronounced activation of type-I ISGs, whereas the upregulation of the NF- $\kappa$ B response gene set is relatively weak. ${ }^{13}$ Hence, the identification and relative activation of NF- $\kappa \mathrm{B}$ and IFN type-I specific genes in MS 


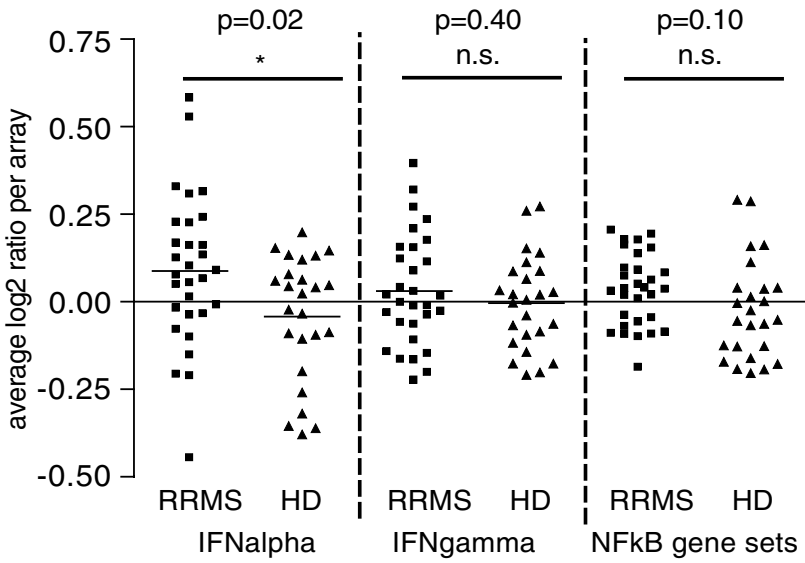

Figure 3 Increased expression of type-I IFN-induced genes in RRMS patients. The average $\log 2$ expression ratio of two IFNspecific (type-I and II) gene sets as well as an NF- $\kappa \mathrm{B}$-specific gene set was calculated for each individual patient and control. The mean expression ratio of the type-I IFN-specific genes was significantly higher in the RRMS patient group when compared to the healthy control group. In contrast, the expression ratio of type-II IFN and $\mathrm{NF}-\kappa \mathrm{B}$ genes did not differ between patients and controls. * is considered significant using an unpaired $t$-test; NS, not significant.

might provide insight into the mechanism of immune activation. In this case, we used the type-I IFN-specific gene set that we have used before, together with an NF$\kappa \mathrm{B}$-specific gene set ( 23 genes) from the Biocarta database (Supplementary data).

In order to confirm and extend the knowledge on the differential gene expression programs that are activated upon differential TLR stimulation, we analyzed previously reported gene expression data sets from experiments in which cells were stimulated with either a bacterial (TLR2 and TLR4) or a viral (TLR3 and TLR7) stimulus. ${ }^{13}$ As expected, we confirmed that bacteria, especially, induce the $\mathrm{NF}-\kappa \mathrm{B}$ gene set, whereas viral agents show a more pronounced induction of the type-I IFN-specific gene set (Supplementary data). To investigate the relative contribution of either gene set in the RRMS gene expression profile, we calculated for both gene sets the mean gene expression level per patient and healthy control and compared the two groups with each other (Figure 3). As described above this analysis showed that the mean gene expression level of the type-I IFN-induced gene set was significantly higher in the MS patient group when compared to the healthy control group. However, the mean gene expression level of the NF- $\kappa \mathrm{B}$-specific genes was similar between patients and controls. The weak expression of NF- $\kappa \mathrm{B}$ genes in combination with the upregulation of type-I IFN genes would argue against a direct contribution of a TLR2- or TLR4-induced activation on PB cells. Therefore, these data suggest that if a microbial infection is directly involved in the PB host response in MS, this is most likely of viral origin.

\section{Gene expression profiles compared to a poxvirus} infection model

Next, we evaluated the relationship between a viral response program and RRMS in more detail, by comparing the gene expression profiles of MS patients with the profiles of an in vivo virus infection model. For that purpose, we used a data set from a non-human primate model for smallpox (SP) infection, which provides a detailed picture of the in vivo host transcriptional response program in $\mathrm{PB}$ cells after a viral infection. ${ }^{16}$ The data were generated on similar human cDNA microarrays that we have used, and was also stored and analyzed using the Stanford Microarray Database (SMD). We extracted microarray data from nine macaques before and 2 or 3 days after infection from the public access website of the SMD. These data were filtered (see Materials and methods) and used to construct a SP virus-induced gene set. We applied SAM analysis to select the significantly differentially expressed genes between the PB gene expression profiles before and after SP infection. The resulting 1307 statistically differential expressed genes (False Discovery Rate (FDR) <5\%) were used for an unsupervised hierarchical clustering of the PB gene expression data from the macaques (before and after infection), RRMS patients and healthy controls (Figure 4). As anticipated, this analysis clearly separated the profiles of the macaques before and after viral infection. In addition, almost all the healthy controls clustered in the same group as the unaffected animals. Most interestingly, the MS patients were clearly divided into two groups on the basis of their gene expression profile. More than $50 \%$ of the RRMS patients clustered together with the virus-infected animals and showed an upregulation of immune defense genes. We refer to this group as the RRMShigh group. The patient who was analyzed twice, clustered together within this group showing the reliability of the analysis. The remaining patients, designated as the RRMS ${ }^{\text {low }}$ group, clustered with the uninfected animals. These results demonstrate a remarkable similarity between the virus-induced gene expression profiles of the macaques and the $\mathrm{PB}$ profile of a significant part $\left(P=0.0002 ; \chi^{2}\right.$ test $)$ of the MS patients.

\section{Heterogeneity of RRMS}

The above analysis revealed a striking heterogeneity between RRMS patients. Analysis of the overall PB profiles of only the RRMS patients using unsupervised hierarchical clustering confirmed the sub-classification of MS patients into two groups. The result of the cluster analysis correlated with the sub-classification based on the SP virus profile (data not shown). These results point towards an important discriminating role for immune defense activity in RRMS. To further investigate the biological basis of RRMShigh group, we compared the expression profiles of only the RRMShigh group of patients with the healthy controls by PANTHER pathway-level analysis. In accordance with the overall RRMS group versus healthy controls comparison (Table 1 and Figure 2) using PANTHER analyses, an upregulation of genes involved in IFN signaling, Alzheimer disease, inflammation, angiogenesis, interleukin signaling, JAK/ STAT signaling, TLR signaling, glycolysis and apoptosis was observed in the RRMShigh group. In addition, this comparison revealed the upregulation of genes involved in the Ras and epidermal growth factor receptor signaling pathway (Table 3). Interestingly, all the pathways that are specific for the RRMS high group overlap with those of the common response pathway from Jenner and Young RRMS high group, ${ }^{13}$ which is in favor of an infectious origin for the immune defense program observed in this specific patient subgroup (Table 2). 


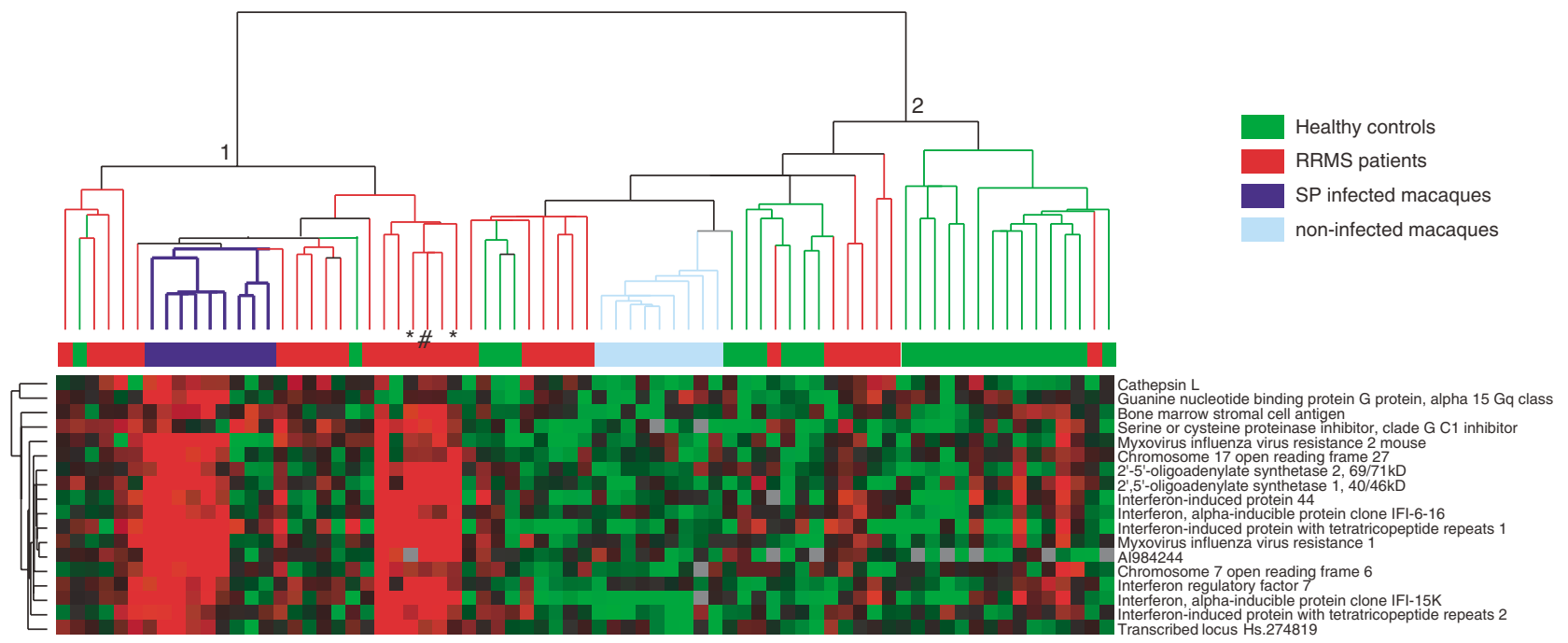

Figure 4 A significant part of the RRMS patients co-clusters with SP-infected macaques. Genes were pre-selected, of which the gene expression levels were significantly (using SAM: FDR $<5 \%$ ) changed in the SP-infected macaques when compared to the non-infected macaques. Subsequently, these genes were used for unsupervised (two-way) hierarchical cluster analysis on the combined data from the SP study and our study. This type of clustering will not only place genes that have correlated expression levels in adjacent rows but also places samples that have similar expression profiles in adjacent columns. A subsequent selection of genes was made, by using only those genes whose gene expression levels were two-fold different from the median expression level in at least four samples. In this figure, only part of the total cluster, representing IFN-related genes, is shown. The patient who was analyzed twice (marked *) clustered together in same arm of the dendogram. The patient receiving IFN- $\beta$ treatment (marked ${ }^{\#)}$ co-clustered with the virus-infected macaques.

Table 3 Pathway-level analyses; RRMShigh group versus healthy controls

Pathways significantly upregulated in RRMS ${ }^{\text {high }}$

Inflammation mediated by chemokine and cytokine signaling pathway

Angiogenesis

IFN- $\gamma$ signaling pathway

Interleukin signaling pathway

JAK/STAT signaling pathway

Toll-receptor signaling pathway

Ras pathway

Alzheimer disease - presenilin pathway

Glycolysis

EGF receptor signaling pathway

Abbreviations: EGF, epidermal growth factor; IFN, interferon; RRMS, relapsing-remitting multiple sclerosis.

Comparison between the RRMS ${ }^{\text {high }}$ patient group and the healthy control group using PANTHER pathway-level analysis. Pathways were considered significant if $P$-value $<0.05$.

GSEA analysis was again used to confirm the results. Interestingly, using this analysis, especially the type-I IFN-induced and viral response gene sets were highly significant enriched in the subgroup of RRMS (data not shown).

Clearly, the molecular heterogeneity that we observed in the PB profiles of RRMS patients is indicative of the heterogeneous nature of RRMS and may suggest the existence of different subtypes of RRMS. The question then arose as to whether the molecular stratification of RRMS was associated with clinical differences between the patients. To address this possibility, the relationship of the subtypes with clinical and demographic parameters was determined. However, we were not able to demonstrate an association between the molecular subtypes and clinical and demographic parameters, such as age, disease duration, relapse rate and expanded disability severity score.

\section{Discussion}

Several investigators studied gene expression levels in $\mathrm{PB}$ cells to address the question, whether disease characteristics are detectable systemically. These previous studies demonstrated changes in PB gene expression patterns with among these, genes involved in the p53 and apoptosis pathway. ${ }^{17-21}$ Whereas these studies provided insight into the genes that were differentially expressed between MS patients and healthy controls, the issue of transcript-based disease heterogeneity was not addressed.

Using pathway analysis, we found next to the p53 and apoptosis pathways several other pathways and biological processes that are significantly different between RRMS patients and healthy controls. The observed upregulation of GPX1 and NCF1 together with the downregulation of catalase is suggestive of a disturbed oxidative stress response in RRMS. Although oxidative stress has been implicated to be involved in virusinduced neuronal injury, 22 our results seem to be in conflict with a recent paper published by Hultqvist et al. ${ }^{23}$ They showed that a mutation in the NCF1 gene, leading to a reduced oxidative burst, enhanced the autoimmune susceptibility for arthritis and encephalomyelitis in animal models. The difference may be owing to a different role of oxidative burst in disease initiation versus established disease.

Another pathway that was upregulated in MS is glycolysis, which is an important metabolic pathway 
for the ATP biosynthesis of cells. Induction of glycolysis is typically observed in virally infected cells. Viruses do not generate the ATP that they require and rely heavily on the host cells for ATP biosynthesis.

Interestingly, we discovered for the first time significantly increased gene expression levels for several genes involved in (type-I) IFN-mediated immunity in at least half of the MS patients. One of these genes is ADAR1 (double-stranded RNA (dsRNA)-dependent adenosine deaminase), which is induced by type-I IFNs and is involved in editing of viral RNAs ${ }^{24}$ and the mRNA of glutamate receptor units. ${ }^{25}$ Other IFN-induced genes that showed upregulated expression levels in RRMS were OAS1, OAS2, MX1 and MX2. The OAS genes encode for dsRNA-dependent synthetases that activate the endoribonuclease RNAse L to degrade single-stranded RNA (ssRNA). ${ }^{26}$ The IFN-inducible Mx proteins are GTPases that interfere with virus replication, probably by inhibiting the trafficking ${ }^{27}$ or activity of virus polymerases. ${ }^{28}$ The increased expression of MxA confirms data from a previous report that also showed increased expression of MxA at the protein level in PB cells of RRMS patients. ${ }^{29}$ Accordingly, Chieux et al. ${ }^{29}$ also showed increased levels of IFN- $\alpha$ in the serum of MS patients.

An upregulation of IFN-induced genes is also observed in other autoimmune diseases like SLE, ${ }^{30}$ rheumatoid arthritis ${ }^{31}$ Sjögren's syndrome (SS), ${ }^{32}$ type I diabetes mellitus ${ }^{33}$ and dermatomyositis. ${ }^{34}$ Moreover, the disease activity of SLE patients was correlated with increased serum levels of IFN- $\alpha .{ }^{35}$ However, for MS, the available clinical data suggest that IFN- $\beta$ is involved in inhibiting the disease symptoms. These findings suggest that type-I IFNs might have differential clinical effects depending on the disease. Although in SLE the pDC in the PB have been identified as a major source of IFN- $\alpha$, in SS numerous IFN- $\alpha$-producing cells were detected in the affected salivary gland biopsies. These studies suggest that in SS the source of IFN can be from cells in the PB blood as well as from cells in the diseased tissue. Further studies are needed to demonstrate the source of IFN in MS.

Additionally, our data analysis showed a coordinated upregulation of genes involved in TLR signaling, indicative of an activated innate immune signaling. In theory, infectious agents or endogenous factors, such as viruses, bacteria, unmethylated CpG DNA or ssRNA or dsRNA, could be proximal mediators of type-I IFN production and more downstream activation programs. Innate immune responses to pathogens are believed to be patterned and stereotyped. The specialization of different TLRs allows the host to tailor its response to different pathogens. This is achieved by the differential activation of at least two different but partly overlapping pathways: the MyD88-dependent and the IRF3/7 MyD88-independent pathway. Both pathways lead to the induction of the $\mathrm{NF}-\kappa \mathrm{B}$ response program and gene activation via the IFN response element, resulting in the transcription of IFNrelated genes. In general, bacterial pathogens and specific endogenous factors such as heat-shock proteins induce a strong $\mathrm{NF}-\kappa \mathrm{B}$ response along with a moderate or weak IFN response. In contrast, other factors such as ssRNA or dsRNA or viruses induce a strong type-I IFN response along with a weak to absent $N F-\kappa B$ response. Our results showed a high expression of type-I IFNinduced genes in MS patients, whereas the expression of
$\mathrm{NF}-\kappa \mathrm{B}$-specific genes was comparable with that of the controls. Thus, if activation of PB cells of MS patients is responsible for the observed activation of IFN-induced genes, it is unlikely that this occurred via the MyD88dependent pathway, as we did not observe a difference in NF- $\kappa \mathrm{B}$-specific gene expression levels. Together with the observation that our data did demonstrate the upregulation of genes involved in TLR and type-I IFNsignaling, this would restrict the activation of IFNinduced genes to TLR receptors that selectively signal via the IRF3/7 pathway, like TLR3, TLR7/TLR8, TLR936 or via the recently discovered RNA helicase RIG-I that bind cytoplasmic dsRNA ${ }^{37}$ or other thus far unknown ligands.

Infectious agents have long been considered as possible triggers for autoimmune responses. The search for a specific virus in MS may be hampered owing to the fact that there might not be a single MS-specific virus, but a variety of distinct viruses or a group of related viruses that are involved. ${ }^{38}$ Upon infection with a pathogen, cells undergo a marked reprogramming of their transcriptome. ${ }^{13}$ Part of the response program has been interpreted as a generic 'alarm signal' to infection and involves functional groups of genes involved in, for example, the IFN response, inflammation, apoptosis and TLR signaling. ${ }^{13}$ Here, we demonstrated that the pathways that were found to be different between healthy controls and RRMS patients overlap for a great part with the pathways that are induced during the common pathogen response program described by Jenner and Young $^{13}$ (Table 1). This overlap is even more convincingly demonstrated in the RRMSigh patient group, which showed a high expression of immune defense and IFN-related genes. Moreover, we observed a striking similarity of the PB gene expression signature in MS with that of the virus-infected macaque model, which demonstrates similarity of gene clusters that constitute a general 'alarm signal' for a viral infection. These findings might suggest that a viral agent could play a role in MS.

However, despite the clear relation of a virus response program and the MS gene signature, for SLE and SS, it was shown that immune complexes from patients IgG together with nucleic acid released by necrotic of apoptotic cells can induce type-I IFN production by PB plasmacytoid DCs and a concomitant type-I IFN response signature in the PB. ${ }^{39}$ Thus, although the data from epidemiological studies and the similarity of the PB gene expression signature in MS with that of the virusinfected macaque model support a role for viruses in MS, we cannot formally exclude a contribution for endogenous factors in the induction of the type-I IFN response program in MS. Hence, further studies are needed to determine whether the increased expression of type-I IFN response genes are MS are the result of endogenous or infectious factors.

Our results clearly indicate that there is a striking difference in gene expression profiles between RRMS patients. The wide variation in clinical symptoms, disease course and therapy response is consistent with this observation. The question is what the molecular classification into RRMS high and RRMS low means in clinical terms. Based on the expression profiles, it suggests that different pathogenic mechanisms may contribute to disease. Therefore, this type of analysis provides a rational to understand the molecular and 
biological basis of disease heterogeneity in MS. So far, we could not demonstrate an association with clinical parameters. However, we realize that the design of this study does not allow any firm conclusions to be drawn.

Because the therapeutic response to IFN- $\beta$ therapy also seems to be heterogeneous, ${ }^{2}$ it is tempting to speculate that either one of the subtypes is more likely to respond better to IFN- $\beta$ treatment. Not so surprisingly, several reports have demonstrated an induction of the type-I IFN response program in patients treated with IFN- $\beta$ .$^{40,41}$ As anticipated, the one patient receiving IFN- $\beta$ therapy clustered together with the RRMSigh patients and showed an upregulation of IFN-induced genes. Although transcription-based prediction of IFN- $\beta$ therapy has been studied, ${ }^{42,43}$ future research in a large cohort of patients is needed to sort out whether the absence or presence of a type-I IFN response in the PB of patients with RRMS is a prognostic factor for successful treatment with IFN- $\beta$.

\section{Materials and methods}

\section{Patients}

Dutch patients with definite $\mathrm{MS}^{44}$ were recruited from the outpatient clinic of the MS Centre at the VUMC Amsterdam. We selected 29 patients with relapsingremitting disease, of which 28 had not been treated with IFN- $\beta$ for at least 3 years. Some of these patients were not clinically active, some had declined disease-modifying drugs (DMDs), and in others, the sample was taken immediately before they started DMDs. Of the 29 patients, 10 patients were included during an acute relapse and one patient who did receive IFN- $\beta$ therapy was included in the analysis. In addition, one patient was analyzed twice. The control group consisted of 25 healthy volunteers, matched with respect to gender and age. All subjects were unrelated and Dutch Caucasians. This study was carried out with the approval of the Medical Ethics Committee of the VUMC, and informed consent was obtained from all subjects.

\section{Blood sampling}

Blood (2.5 ml) was drawn in PAXgene blood RNA isolation tubes (PreAnalytix, GmbH, Germany). After blood collection, the tubes were incubated for $2 \mathrm{~h}$ at room temperature (RT) to ensure complete lyses of all the blood cells, after which the tubes were stored at $-20^{\circ} \mathrm{C}$. Total RNA was isolated within 7 months after blood collection. Tubes were thawed for $2 \mathrm{~h}$ at RT before RNA isolation. Next, total RNA was isolated using the PAXgene RNA isolation kit according to the manufacturers' instructions, including a DNAse (Qiagen, Venlo, The Netherlands) step to remove genomic DNA.

\section{Sample preparation for array hybridization}

Sample preparation and array hybridization was performed as described previously. ${ }^{45}$ Total RNA was linear amplified using the Message Amp. aRNA kit (Ambion, Huntingdon, UK), which is based on the Eberwine method. ${ }^{46}$ We used $1 \mu \mathrm{g}$ of total RNA as input and followed the protocol according to the manufacturer's instructions allowing $5 \mathrm{~h}$ for in vitro transcription.

Per array hybridization, two separate reaction vials were set up for labeling: one for the experimental sample to be labeled with $\mathrm{Cy} 3$ (green) and one for the common reference sample to be labeled with Cy5 (red). We made a common reference that consisted of a mixture of mRNAs isolated from 11 different cell lines, ${ }^{47}$ supplemented with RNA from rheumatoid synovial tissue, fibroblasts and activated peripheral blood mononuclear cells. From both samples, $5 \mu \mathrm{g}$ aRNA was labeled with aminoallyl-dUTP during cDNA synthesis by reverse transcriptase, followed by chemical coupling of the aminoallyl group to $\mathrm{Cy} 3$ or $\mathrm{Cy} 5$ for the experimental and reference samples, respectively.

The labeled cDNA transcripts of an MS patient or healthy control and a common reference are then hybridized together onto the $43 \mathrm{~K}$ cDNA microarrays (Stanford University) at $65^{\circ} \mathrm{C}$. After $18 \mathrm{~h}$ of hybridization and multiple washing steps, the hybridized cDNA products are measured by fluorescent colorimetry using the G2505B microarray scanner from Agilent Technologies (Amstelveen, The Netherlands).

Data filtering and analysis

The array images were analyzed using the program GenePix Pro 3.0 (Axon instruments Inc., Union City, CA, USA) and all data were uploaded and analyzed using the Stanford Mircroarray Database (SMD) (http:// smd.stanford.edu). ${ }^{48}$ Intensity-dependent normalization using local estimation ${ }^{49}$ ('Loess') was performed separately on each sector of the array ('stratified' by printtip). In addition, 'scale' normalization was applied, which makes the data more comparable across different arrays. Next, we excluded data from spots with inconsistent hybridization signals (defined by a regression $R^{2}<0.6$ for a linear fit between the Cy3 and Cy5 pixel intensities) or spots that had been flagged as defective by visual inspection during data extraction (for instance, owing to scratches or dust particles). In addition, we only included spots if the signal intensity was 2.5 times higher than the background and less than the saturated value of 65000 . We corrected for array batch differences by applying single value decomposition. Because a single gene can be represented more than once on an array, we collapsed our data by averaging the results from sequences with the same identifier (Unigene Cluster ID) in the database. Finally, we re-filtered our data in the SMD and median centered the data for genes and arrays.

We used the $\log 2$ of the background-subtracted, normalized ratio of the mean $\mathrm{Cy} 5$ and mean Cy3 expression values and applied several algorithms (see flowchart in Figure 1) for the analysis. For all algorithms, we only used data from arrays if there was at least $80 \%$ good data present per gene. SAM was used ${ }^{9}$ to determine the significantly differentially expressed genes between different subject groups. We considered genes as significantly differential expressed, if the FDR was less than $5 \%$ (a $q$-value of less than 0.05 ). Cluster ${ }^{50}$ was used to define clusters of coordinately regulated genes after which Treeview visualized the clustered data.

Additionally, we applied PANTHER (PE Applied Biosystems, Foster City, CA, USA; http://PANTHER.appliedbiosystem.com ${ }^{51}$ ) to interpret our data after SAM analysis. This analysis is based on a binomial distribution test to classify the list of significantly up- or downregulated genes (referred to as 'SAM gene list') in different functional categories (molecular function, biological process or biological pathway). After classifica- 
tion, a significant $P$-value $(P<0.05)$ indicates that a given category may be of biological interest.

\section{Pathway-level analysis in order to find coordinated shifts in gene expression levels}

We used two different algorithms, both designed to find weak coordinated shifts in gene expression data: PANTHER (Applied Biosystems) and GSEA ${ }^{12}$ (GSEA).

For the PANTHER pathway-level analysis, we used all differentially expressed genes with their associated fold changes between two groups, for example, RRMS patients and healthy controls. The Mann-Whitney $U$-test is used to look for coordinated changes in gene expression within each available pathway. As a result, a pathway may be found significant even if the fold changes are very small.

In contrast to the PANTHER analysis, the pathwaylevel analysis program GSEA uses the total raw data file consisting of the normalized ratio's per gene per array. GSEA combines a priori defined sets of genes to increase signal-to-noise ratio and improve statistical power to determine if genes of a given gene set are enriched among the most differentially expressed genes between patients and healthy controls. We applied GSEA as described before, ${ }^{52}$ only to confirm the PANTHER analysis; therefore, the data are not shown.

\section{Acknowledgements}

This study was partly supported by the Centre for Medical Systems Biology (a center of excellence approved by the Netherlands Genomics Initiative/Netherlands Organization for Scientific Research), and grants from the Stichting MS Research. We would like to thank the following authors for making their microarray data publicly available: RG Jenner (Cambridge, USA), KH Rubins (Stanford, USA) and Q Huang (Cambridge, USA).

\section{References}

1 Hafler DA. Multiple sclerosis. J Clin Invest 2004; 113: 788-794.

2 Rio J, Nos C, Tintore M, Borras C, Galan I, Comabella M et al. Assessment of different treatment failure criteria in a cohort of relapsing-remitting multiple sclerosis patients treated with interferon beta: implications for clinical trials. Ann Neurol 2002; 52: 400-406.

3 Lucchinetti C, Bruck W, Parisi J, Scheithauer B, Rodriguez M, Lassmann H. Heterogeneity of multiple sclerosis lesions: implications for the pathogenesis of demyelination. Ann Neurol 2000; 47: 707-717.

4 Bielekova B, Kadom N, Fisher E, Jeffries N, Ohayon J, Richert $\mathrm{N}$ et al. MRI as a marker for disease heterogeneity in multiple sclerosis. Neurology 2005; 65: 1071-1076.

5 Sadovnick AD, Armstrong H, Rice GP, Bulman D, Hashimoto L, Paty DW et al. A population-based study of multiple sclerosis in twins: update. Ann Neurol 1993; 33: 281-285.

6 Pugliatti M, Sotgiu S, Rosati G. The worldwide prevalence of multiple sclerosis. Clin Neurol Neurosurg 2002; 104: 182-191.

7 Kurtzke JF. Epidemiology of MS. In: Hallpike JF, Adams CWM, Tourtellote WE (eds). Multiple Sclerosis. Willimas and Wilkins: Baltimore, MD, 2005, pp. 49-95.

8 Ter Meulen V, Katz M. The proposed viral etiology of multiple sclerosis and related demyelinating diseases. In: Raine CS,
MacFarland HF, Tourtellotte WW (eds). Multiple Sclerosis Chapman \& Hall: London, 1997, pp. 287-305.

9 Tusher VG, Tibshirani R, Chu G. Significance analysis of microarrays applied to the ionizing radiation response. Proc Natl Acad Sci USA 2001; 98: 5116-5121.

10 Cho RJ, Campbell MJ. Transcription, genomes, function Trends Genet 2000; 16: 409-415.

11 Mi H, Lazareva-Ulitsky B, Loo R, Kejariwal A, Vandergriff J, Rabkin $S$ et al. The PANTHER database of protein families, subfamilies, functions and pathways. Nucleic Acids Res 2005; 33: D284-D288.

12 Subramanian A, Tamayo P, Mootha VK, Mukherjee S, Ebert BL, Gillette MA et al. Gene set enrichment analysis: a knowledge-based approach for interpreting genomewide expression profiles. Proc Natl Acad Sci USA 2005; 102: 15545-15550.

13 Jenner RG, Young RA. Insights into host responses against pathogens from transcriptional profiling. Nat Rev Microbiol 2005; 3: 281-294.

14 Samuel CE. Antiviral actions of interferons. Clin Microbiol Rev 2001; 14: 778-809, Table.

15 Moynagh PN. TLR signalling and activation of IRFs: revisiting old friends from the NF-kappaB pathway. Trends Immunol 2005; 26: 469-476.

16 Rubins KH, Hensley LE, Jahrling PB, Whitney AR, Geisbert TW, Huggins JW et al. The host response to smallpox: analysis of the gene expression program in peripheral blood cells in a nonhuman primate model. Proc Natl Acad Sci USA 2004; 101 15190-15195.

17 Achiron A, Gurevich M, Friedman N, Kaminski N, Mandel M. Blood transcriptional signatures of multiple sclerosis: unique gene expression of disease activity. Ann Neurol 2004; 55: 410-417.

18 Aune TM, Maas K, Moore JH, Olsen NJ. Gene expression profiles in human autoimmune disease. Curr Pharm Des 2003; 9: 1905-1917.

19 Maas K, Chan S, Parker J, Slater A, Moore J, Olsen N et al. Cutting edge: molecular portrait of human autoimmune disease. J Immunol 2002; 169: 5-9.

20 Mandel M, Gurevich M, Pauzner R, Kaminski N, Achiron A. Autoimmunity gene expression portrait: specific signature that intersects or differentiates between multiple sclerosis and systemic lupus erythematosus. Clin Exp Immunol 2004; 138: 164-170.

21 Satoh J, Nakanishi M, Koike F, Miyake S, Yamamoto T, Kawai $\mathrm{M}$ et al. Microarray analysis identifies an aberrant expression of apoptosis and DNA damage-regulatory genes in multiple sclerosis. Neurobiol Dis 2005; 18: 537-550.

22 Valyi-Nagy T, Dermody TS. Role of oxidative damage in the pathogenesis of viral infections of the nervous system. Histol Histopathol 2005; 20: 957-967.

23 Hultqvist M, Olofsson P, Holmberg J, Backstrom BT, Tordsson J, Holmdahl R. Enhanced autoimmunity, arthritis, and encephalomyelitis in mice with a reduced oxidative burst due to a mutation in the Ncf1 gene. Proc Natl Acad Sci USA 2004; 101: 12646-12651.

24 Patterson JB, Thomis DC, Hans SL, Samuel CE. Mechanism of interferon action: double-stranded RNA-specific adenosine deaminase from human cells is inducible by alpha and gamma interferons. Virology 1995; 210: 508-511.

25 Lomeli H, Mosbacher J, Melcher T, Hoger T, Geiger JR, Kuner $\mathrm{T}$ et al. Control of kinetic properties of AMPA receptor channels by nuclear RNA editing. Science 1994; 266: 1709-1713.

26 Player MR, Torrence PF. The 2-5A system: modulation of viral and cellular processes through acceleration of RNA degradation. Pharmacol Ther 1998; 78: 55-113.

27 Reichelt M, Stertz S, Krijnse-Locker J, Haller O, Kochs G. Missorting of LaCrosse virus nucleocapsid protein by the interferon-induced MxA GTPase involves smooth ER membranes. Traffic 2004; 5: 772-784. 
28 Stranden AM, Staeheli P, Pavlovic J. Function of the mouse $\mathrm{Mx} 1$ protein is inhibited by overexpression of the PB2 protein of influenza virus. Virology 1993; 197: 642-651.

29 Chieux V, Chehadeh W, Hautecoeur P, Harvey J, Wattre P, Hober D. Increased levels of antiviral MxA protein in peripheral blood of patients with a chronic disease of unknown etiology. J Med Virol 2001; 65: 301-308.

30 Baechler EC, Batliwalla FM, Karypis G, Gaffney PM, Ortmann $\mathrm{WA}$, Espe $\mathrm{KJ}$ et al. Interferon-inducible gene expression signature in peripheral blood cells of patients with severe lupus. Proc Natl Acad Sci USA 2003; 100: 2610-2615.

31 van der Pouw Kraan CTM, Wijbrandts CA, van Baarsen LGM, Voskuyl AE, Rustenburg F, Baggen JM et al. Rheumatoid arthritis subtypes identified by genomic profiling of peripheral blood cells: assignement of a type I interferon signature in a subpopulation of patients (submitted for publication).

32 Bave U, Nordmark G, Lovgren T, Ronnelid J, Cajander S, Eloranta ML et al. Activation of the type I interferon system in primary Sjogren's syndrome: a possible etiopathogenic mechanism. Arthritis Rheum 2005; 52: 1185-1195.

33 Huang X, Yuang J, Goddard A, Foulis A, James RF, Lernmark A et al. Interferon expression in the pancreases of patients with type I diabetes. Diabetes 1995; 44: 658-664.

34 Greenberg SA, Pinkus JL, Pinkus GS, Burleson T, Sanoudou D, Tawil $\mathrm{R}$ et al. Interferon-alpha/beta-mediated innate immune mechanisms in dermatomyositis. Ann Neurol 2005; 57: 664-678.

35 Bengtsson AA, Sturfelt G, Truedsson L, Blomberg J, Alm G, Vallin $\mathrm{H}$ et al. Activation of type I interferon system in systemic lupus erythematosus correlates with disease activity but not with antiretroviral antibodies. Lupus 2000; 9: 664-671.

36 Akira S, Takeda K. Toll-like receptor signalling. Nat Rev Immunol 2004; 4: 499-511.

37 Yoneyama M, Kikuchi M, Natsukawa T, Shinobu N, Imaizumi $\mathrm{T}$, Miyagishi $\mathrm{M}$ et al. The RNA helicase RIG-I has an essential function in double-stranded RNA-induced innate antiviral responses. Nat Immunol 2004; 5: 730-737.

38 van Noort JM, Bajramovic JJ, Plomp AC, van Stipdonk MJ. Mistaken self, a novel model that links microbial infections with myelin-directed autoimmunity in multiple sclerosis. J Neuroimmunol 2000; 105: 46-57.

39 Lovgren T, Eloranta ML, Bave U, Alm GV, Ronnblom L. Induction of interferon-alpha production in plasmacytoid dendritic cells by immune complexes containing nucleic acid released by necrotic or late apoptotic cells and lupus IgG. Arthritis Rheum 2004; 50: 1861-1872.

40 Weinstock-Guttman B, Badgett D, Patrick K, Hartrich L, Santos R, Hall D et al. Genomic effects of IFN-beta in multiple sclerosis patients. I Immunol 2003; 171: 2694-2702.
41 Koike F, Satoh J, Miyake S, Yamamoto T, Kawai M, Kikuchi S et al. Microarray analysis identifies interferon beta-regulated genes in multiple sclerosis. J Neuroimmunol 2003; 139: 109-118.

42 Baranzini SE, Mousavi P, Rio J, Caillier SJ, Stillman A, Villoslada $\mathrm{P}$ et al. Transcription-based prediction of response to IFNbeta using supervised computational methods. PLoS Biol 2005; 3: e2

43 Sturzebecher S, Wandinger KP, Rosenwald A, Sathyamoorthy M, Tzou A, Mattar P et al. Expression profiling identifies responder and non-responder phenotypes to interferon-beta in multiple sclerosis. Brain 2003; 126: 1419-1429.

44 McDonald WI, Compston A, Edan G, Goodkin D, Hartung HP, Lublin FD et al. Recommended diagnostic criteria for multiple sclerosis: guidelines from the International Panel on the diagnosis of multiple sclerosis. Ann Neurol 2001; 50: 121-127.

45 van der Pouw Kraan TC, Baarsen EGM, Rustenburg F et al. Gene expression profiling in rheumatology. In: Cope A (ed). Methods in Molecular Medicine. The Humana Press Inc.: Totowa, NJ, 2005.

46 Van Gelder RN, von Zastrow ME, Yool A, Dement WC, Barchas JD, Eberwine JH. Amplified RNA synthesized from limited quantities of heterogeneous cDNA. Proc Natl Acad Sci USA 1990; 87: 1663-1667.

47 Perou CM, Jeffrey SS, van de RM, Rees CA, Eisen MB, Ross DT et al. Distinctive gene expression patterns in human mammary epithelial cells and breast cancers. Proc Natl Acad Sci USA 1999; 96: 9212-9217.

48 Ball CA, Awad IA, Demeter J, Gollub J, Hebert JM, Hernandez-Boussard $\mathrm{T}$ et al. The standard microarray database accommodates additional microarray platforms and data formats. Nucleic Acids Res 2005; 33 (Database issue): D580-D582.

49 Yang YH, Dudoit S, Luu P, Lin DM, Peng V, Ngai J et al. Normalization for cDNA microarray data: a robust composite method addressing single and multiple slide systematic variation. Nucleic Acids Res 2002; 30: e15.

50 Eisen MB, Spellman PT, Brown PO, Botstein D. Cluster analysis and display of genome-wide expression patterns. Proc Natl Acad Sci USA 1998; 95: 14863-14868.

51 Thomas PD, Campbell MJ, Kejariwal A, Mi H, Karlak B, Daverman $\mathrm{R}$ et al. PANTHER: a library of protein families and subfamilies indexed by function. Genome Res 2003; 13: 2129-2141.

52 Mootha VK, Lindgren CM, Eriksson KF, Subramanian A, Sihag S, Lehar J et al. PGC-1alpha-responsive genes involved in oxidative phosphorylation are coordinately downregulated in human diabetes. Nat Genet 2003; 34: 267-273.

Supplementary Information accompanies the paper on Genes and Immunity website (http://www.nature.com/gene) 